\title{
Modeling and Velocity Tracking Control for Tape Drive System
}

\section{*11OKUBANJO, A.A; ${ }^{1}$ OYETOLA, O.K; ${ }^{1}$ OSIFEKO, M.O ${ }^{2}$ ADEKOMAYA, S.O}

Department of Computer and Electrical Electronics Engineering, ${ }^{2}$ Department of Mechanical Engineering, Faculty of Engineering, Olabisi Onabanjo University, Ibogun Campus, Ogun, Nigeria.

\begin{abstract}
The objectives of this paper are to formulate mathematical model for the tape drive system (TDS) by designing velocity tracking controller using physical laws. A Matlab function script and Simulink model were developed in Matlab and Simulink environment. The result of the study revealed that 7.07, 8 and 10 of $\mathrm{k}_{\text {oln }}$ values met the design goal and also resulted in optimal control performance with the following characteristics $7.31 \%, 7.71 \%$ , 9.41\% overshoot, $1.07,1.08,1.09 \mathrm{sec}$ peak time , $0.385,0.346,0.281 \mathrm{sec}$ settling time, 149,168 and 210 proportional gain, and 67.2,65.6, 62.3 phase margin respectively. While the responses at other values of koln resulted in an unsatisfactory performance. Based on simulation results and observations, it is evident that prediction of the performance of the controller depends on numerical value of $\mathrm{k}_{\text {oln }}$.More so, larger values of $\mathrm{k}_{\text {oln }}$ lead to substantial increase in velocity variation resulting into worst prediction of the controller parameters.
\end{abstract}

DOI: https://dx.doi.org/10.4314/jasem.v22i3.8

Copyright: Copyright $\odot 2018$ Okubanjo et al. This is an open access article distributed under the Creative Commons Attribution License (CCL), which permits unrestricted use, distribution, and reproduction in any medium, provided the original work is properly cited.

Dates: Received: 07 February 2018; Revised: 02 March: 2018; Accepted: 23 March 2018

Keywords: Tape drive system; modeling; control; Matlab and Simulink model

\begin{tabular}{|c|c|c|c|c|c|}
\hline \multicolumn{6}{|c|}{ Nomenclatures } \\
\hline$v_{1}$ & Input tape velocity & {$[\mathrm{m} / \mathrm{s}]$} & $H_{c}$ & PI Controller & {$[-]$} \\
\hline$v_{2}$ & Desired tape Velocity & {$[\mathrm{m} / \mathrm{s}]$} & $e$ & Electrical signal & {$[-]$} \\
\hline$\omega_{1}$ & Transmission Angular Velocity & {$[\mathrm{rad} / \mathrm{sec}]$} & $x$ & Displacement & {$[\mathrm{m}]$} \\
\hline $\boldsymbol{k}_{\mathrm{s}}$ & Spring Constant & {$[\mathrm{N} / \mathrm{m}]$} & $\boldsymbol{k}_{t}$ & Integral gain & {$[-]$} \\
\hline$c_{p}$ & Potentiometer Constant & {$[\mathrm{V} / \mathrm{m}]$} & $k_{2}$ & Drive gain & {$[-]$} \\
\hline$\dot{E}_{t}$ & Tacho Signal & {$[\mathrm{V}]$} & $T_{m}$ & Torque developed by motor 2 & {$[\mathrm{Nm}]$} \\
\hline $\boldsymbol{E}_{m}$ & Armature voltage & {$[V]$} & $J_{m}$ & Motor equivalent inertia & {$\left[\mathrm{kgm}^{2}\right]$} \\
\hline
\end{tabular}

The adoption of information and communication technology in all human endeavor had engendered an unprecedented generation of a high volume of data. Consequently, researchers have focused on areas such as data mining, big data, database management system, data modeling and data storage in recent times. Although data storage is considered as the most prominent of all, it has not been well exploited. Technologies such as USB, magnetic tape drive, HDD, diskette etc. are common tools used for storing data. However, tape drive systems have been proven to be more effective for disaster recovery and stable depository (Biskeborn et al., 2018; Angeliki Pantazi and Lantz, 2014). As the amount of data created, replicated and archived are significantly increasing globally (Pantazi et al., 2012), the demand for economical and durable storage solutions is expeditiously growing. Hence, the promising features of low- cost, long- time storage reliable, energy saving capability and huge volumetric capacity has been advantages of the tape drive system over other storage technologies (Cherubini et al., 2016; Pantazi et al., 2012). Generally, tape drive systems are used for data back-ups and restore , instrument recorders (Panda and Engelmann, 2003), archive data and protect crucial information and mass data storage (Panda and Laverenz, 2003) . In a generic TDS, the tape velocity is estimated at the tape head from a servo signal during read/write operations, which exposes the system to disturbances that significantly affect the track density. Variation in the tape velocity and tension dominantly affect TDS performance. Therefore, it is imperative to minimize the variations as much as possible. This necessitates velocity track-follow controller to ensure that the output velocity track the input velocity (servo system) while maintaining a tape tension whose value can be adjusted by the potentiometer voltage.

Modeling and control of tape drive system had received remarkable attention in the field of mechatronics over the past few decades and the quest for new improvement of tape drive tracking velocity control still continues. A dual feedback and feedforward control strategies to improve the trackfollowing performance of the tape head positioning system is proposed by Zhong et al., 2011. The Zhong and coworker concluded that the proposed controllers expedite accurate positioning of the head assembly over the desired track and consequently provide a better tracking control over a single feedback control scheme. 
Also, many authors had proposed various trackfollowing servo system control strategies to reduce disturbances and to increase track density(Boettcher et al., 2009; Cherubini, Furrer, and Lantz 2016; Garifi, Pao, and Touri 2017). Several control algorithms to minimize the damage of TDS from lateral tape motion has been suggested in many literatures(Boettcher et al. 2009; Kim et al., 2010) Furthermore, a simplified mathematical model and control of the tape system with position tension adjustment and velocity tension mode of operation has been designed and implemented in (Cherubini et al., 2016; Garifi et al., 2017; Pantazi et al., 2010). (Panda and Engelmann, 2003; Panda and Laverenz, 2003a). Similarly, Raymond and coworkers presented an adaptive control system to regulate changes in disturbances dynamics of a tape system. In the paper, Youla-Kucera parameterization and position error signal are combined to achieve disturbance rejection in real time de Callafon and Wang, "Adaptive Regulation of Time Varying Disturbances in a Tape Storage System*." . (de Callafon and Wang, 2013), presented a p-type feedback controller dependent on longitudinal tape position in controlling tape drive velocity and tape tension. Recently, Garifi, Pao, and Touri, "Model Predictive Control for Track Following and Disturbance Rejection in a Tape Drive System. This Work Was Supported in Part by the US National Science Foundation (NSF Grant CMMI1234980), and the Hanse-Wissenschaftskolleg Institute for Advanced Study.", presented a disturbance prediction method based on the wavelet denoising technique and system identification and further formulated a model predictive control (MPC) to improve the track following and disturbance rejection. In another study, a robust control, $H_{\infty}$ and feedback scheme for tape tension are presented. The authors further estimated the velocity measurement from three different sources contrary to the conventional method of tape head measurement. The objectives of this work are to formulate a mathematical model of the tape drive system and accurately track the input velocity with the desired velocity and to maintain the tape tension irrespective of the velocity changes.

System Description: Fig.1. shows a schematic view of TDS. The input of the tape is driven by motor 1 and $v_{1}$ is the uncontrollable tape input velocity. The main goal of the control unit is to allow $v_{2}$ track the input velocity and to maintain a tape tension whose value can be adjusted by the voltage $e_{1}$. The tape tension is presumed to be zero after motor 2 , so that motor 2 delivers the tape tension. More so, the system is to be designed such that the velocitytracking controller parameters $k_{p}, k_{t}$ and $\tau_{i}$ fulfilled the design specification as follows:

Phase margin, $P . M \geq 60^{\circ}$ and

Acceleration constant, $k_{a} \geq 25$.

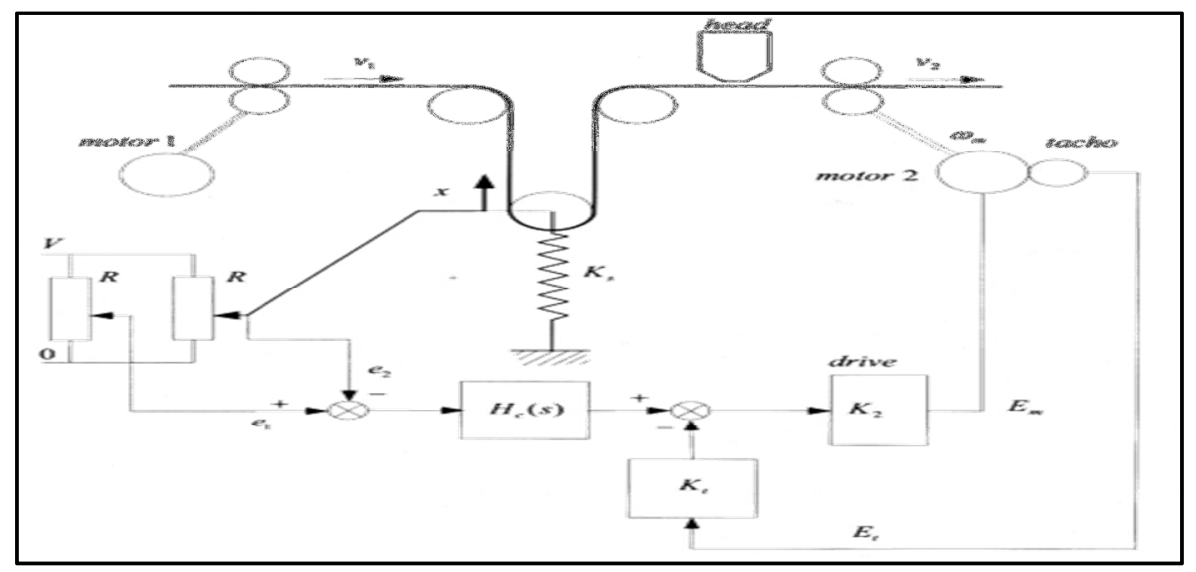

Fig1: schematic overview of tape drive system 


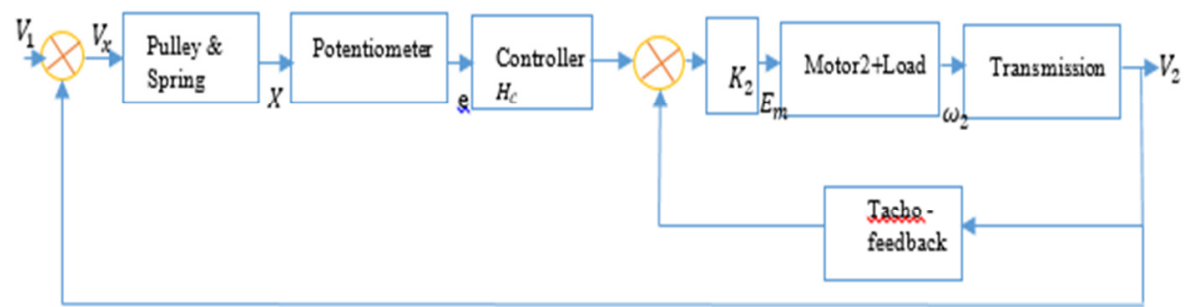

Fig2: schematic block diagram of tape drive system

Mathematical Modelling: The tape drive system was modeled by forming a set of equations for each subsystem as shown in Fig.2 and are further converted to s-domain to simulate the dynamic accurately. The formulation of the mathematical model is considered crucial in this research because the control strategy is examined based on these derived dynamics equations, hence the model must be accurately predicted to represent the dynamic behaviour of the tape drive system. The control procedure is expanded on the derived mathematical model to track the input velocity, hence, we further design a PI controller to track the input velocity. Simulation studies based on MATLAB and Simulink are performed on the tape drive system taken into the consideration the obtained PI controller parameters which are used to validate the mathematical model. The evaluation of the results obtained is presented and discussed extensively concerning achievement as well as providing recommendations for further work. In the model, the following assumptions are made:

That the tape-drive system is fed with a constant angular velocity $\omega$ which initialize a rotational motion with a speed $v_{1}$ in the tape and the speed $v_{2}$ of the motor 2 is measured as output. The input $v_{1}$ is an input signal and $\mathrm{v}_{2}$ as the output signal. The displacement $\mathrm{x}$ of the pulley and spring system depend on the difference in the speed of the two motors $\left(x f\left(v_{1}, v_{2}\right)\right.$; that is to say that a change in the speed produces corresponding to displacement. A reference point (equilibrium point) is taken for the measurement of the displacement $\mathrm{x}$.

When $\mathrm{v}_{\mathrm{x}}=\mathrm{v}_{1}-\mathrm{v}_{2}>0$, then $\mathrm{x}<0$

Equations were derived for each subsystem in sdomain and cascaded to form the overall Transfer function of the system.

For Pulley and spring subsystem: By inspection the cumulative tape distance between the two motors is half the distance of $x$. Mathematically, the change in the cumulative tape distance is twice the displacement $x$ $\int \frac{\partial x}{\partial t}=-2 x$

Recall that $\frac{\partial x}{\partial t}=v_{x}$

Substituting Eq. (2) Into Eq. (1) and take the Laplace result in

$$
\frac{X(s)}{V_{s}(s)}=\frac{-1}{2 s}
$$

For Potentiometer subsystem: The displacement $x$ result in electrical signal

$e=e_{1}-e_{2}$

From the equilibrium point of view, when the system is in equilibrium position, there is no displacement

(since $x=0$ ) and as a result there is no corresponding electrical signal $e=0$

Hence,

$x=C_{p} . x$

Substituting the given value of $C_{p}$ and taking the Laplace transform Eq. (5) yields,

$\frac{E(s)}{X(s)}=-100$

For $\mathrm{H}_{C}:$ PI Controller:

$H_{c}(j \omega)=k \frac{1+j \omega \tau_{i}}{j \omega \tau_{i}}$

For Motor2 + Load subsystem:

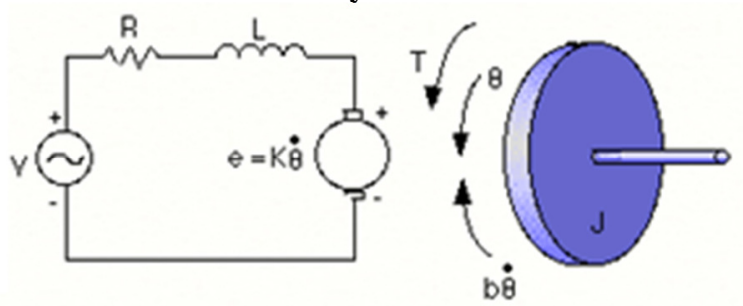

Fig 3: DC motor +load schematic diagram 
magnetic field, its voltage is proportional to speed.

Thus,

$v_{b}=k_{b} \frac{\partial \theta_{m}}{\partial t}$

Since,

$\frac{\partial \theta_{m}(t)}{\partial t}=\omega_{m}(t)$

Putting Eq. (8)into Eq. (9) and taking Laplace transform yields,

$$
V_{b}(s)=K_{b}(s) \omega_{m}(s)
$$

The equation that relate the armature current, $i_{a}(t)$, the applied armature voltage, $e_{a}(t)$, and the back e.m.f $V_{b}(t)$, is found by writing a Kirchhoff's voltage law around the armature circuit in Fig.3

$$
R_{a} i_{a}(t)+L_{a} \frac{\partial i_{a}(t)}{\partial t}+V_{b}(t)=E_{m}(t)
$$

Laplace transform equation (11) yields;

$$
R_{a} I_{a}(s)+s L_{a} I_{a}(s)=E_{m}(s)
$$

The torque developed by the motor 2 is proportional to the armature current, thus,

$$
T_{m}(s)=K_{t} I_{a}(s)
$$

Substituting Eq.13 into Eq.14 yields,

$$
\frac{\left(R_{a}+L_{a} s T_{m}\right) s}{K_{t}}+K_{b} \omega_{m}(s)=E_{m}(s)
$$

$D_{m}$ is the equivalent viscous damping at the armature and includes both the armature viscous damping as well as the load viscous damping reflected to the armature.

$T_{m}(t)+J_{m} \frac{\partial \omega_{m}(t)}{\partial t}+D_{m}(t)$

Hence, substituting Eq. (15) into (14) yields,

$$
\frac{\left(R_{a}+L_{a} s\right)\left(J_{m} s \omega_{m}(s)+D_{m} \omega_{m}(s)\right)}{K_{t}}+K_{b}(s) \omega_{m}(s)=E_{m}(s)
$$

Neglecting armature inductance, the equation becomes;

$$
\frac{\omega_{m}(s)}{E_{m}(s)}=\frac{\frac{K_{t}}{R_{a} J_{m}}}{\left[s+\frac{\left(D_{m}+\frac{K_{t} K_{b}}{R_{a}}\right)}{J_{m}}\right]}
$$

The expression is further simplified as first order system Transfer Function as:

$$
\frac{\omega_{m}(s)}{E_{m}(s)}=\frac{K_{m}}{\left(s+\alpha_{m}\right)}
$$

Where, $K_{m}=\frac{K_{t}}{R_{a} J_{m}}$ and $\alpha_{m}=\frac{\left(D_{m}+\frac{K_{t} K_{b}}{R_{a}}\right)}{J_{m}}$ The parameter values given in Table are substituted in Eq.17 to obtain the motor and load Transfer function as:

$$
\frac{\omega_{m}(s)}{E_{m}(s)}=\frac{5.26}{1+5.26 s}
$$

\section{For Transmission subsystem:}

The relationship between transmission angular velocity and speed can be expressed mathematically as:

$$
\frac{V_{1}(s)}{\omega_{1}(s)}=\frac{V_{2}(s)}{\omega_{2}(s)}
$$

Hence, after substituting the values of the parameter in Tabe. 1 gives

$$
V_{2}=\frac{3 \pi}{20} \mathrm{~m} / \mathrm{s}
$$

\section{For Tachometer subsystem:}

The tachometer output voltage is proportional to angular velocity, which can be expressed mathematically as:

$$
\frac{E_{1}(s)}{\omega_{1}(s)}=\frac{E_{t}(s)}{\omega_{2}(s)}
$$

Similarly, parameter values in table.1 gives,

$$
E_{t}=1 \times \frac{3 \pi}{20} \mathrm{~m} / \mathrm{s}
$$

System overall Transfer Function: The controller parameter values are evaluated from the open loop transfer function, $H_{O L}(s)$ by cascading all the subsystem transfer function as depicted in Fig.4.

$$
H_{O L}(s)=\frac{100 K_{p}}{2 s} \frac{1+\tau_{i} s}{\tau_{i} s}\left[\frac{20 \frac{5.26}{1+5.26 s} \frac{3}{20 \pi}}{1+20 \frac{5.26}{1+5.26 s} \frac{3 K_{t}}{20 \pi}}\right]
$$




$$
\begin{aligned}
& H_{O L}(s)=50 K_{p} \frac{1}{s} \frac{1+\tau_{i} s}{\tau_{i} s}\left[\frac{3}{\pi} \frac{5.26}{(1+5.26 s)} \frac{\pi(1+5.26 s)}{(1+5.26 s \pi)+5.26\left(3 K_{t}\right)}\right] \\
& H_{O L}(s)=\frac{150}{\pi} K_{p} \frac{1+\tau_{i} s}{\tau_{i} s} \frac{1}{s}\left[\frac{5.26}{\left(1+5.26 \frac{3}{\pi} K_{t}\right)+5.26 s}\right]
\end{aligned}
$$

The expression in Eq. (22) is factorized and rearrange to conform to servo system standard transfer function given in Eq. (24) and compared to determine the parameters values.

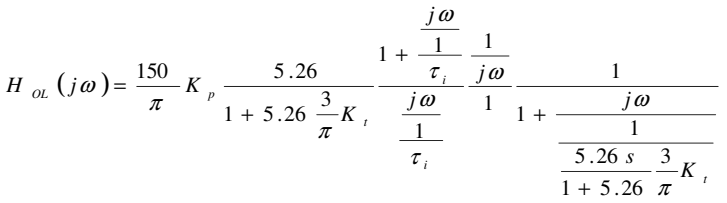

$H_{o L}(s)=K_{o L} \frac{1+j \frac{\omega}{\omega_{1}}}{j \frac{\omega}{\omega_{1}}} \frac{1}{j \frac{\omega}{\omega_{3}}} \frac{1}{1+j \frac{\omega}{\omega_{4}}}$

$$
\begin{aligned}
& K_{O L}=\frac{150}{\pi} K_{p} \frac{5.26}{1+5.26 \frac{3}{\pi} K_{t}}=\frac{251.15 K_{p}}{1+5.02 K_{t}} \\
& K_{p}=\frac{1+5.02 K_{t} K_{O L}}{251.15} \\
& \omega_{1}=\frac{1}{\tau_{i}}, \omega_{3}=1 \\
& \omega_{4}=0.19+0.95 K_{t}
\end{aligned}
$$

According to the servo system bode plot standard form shown in Fig.6 and the system is well tuned if

$$
\omega_{1} \approx \omega_{2} \approx \omega_{3} \& \omega_{4} \gg \omega_{3}
$$

Then, the turning frequencies are computed based on the design requirement and the tuning condition as:

$K_{a}=\omega^{2}=\omega_{1} \omega_{2}=25$

$\omega_{3}=2 \omega_{1}$ $\omega_{4}=20 \omega_{3}$

Hence, tuning frequencies are:

$$
\left\{\begin{array}{l}
\omega_{1}=3.54 \\
\omega_{2}=5 \\
\omega_{3}=7.07 \\
\omega_{4}=141.4
\end{array}\right.
$$

For tuning of the system, $\omega_{3}$ is changed from numerical value of 1 to 7.07, therefore, there is need to express the gain $K_{O L}$ as new gain $\left(K_{O L n}\right)$ and this value is substituted for KOL in Eq. (25)

$$
K_{O L N}=\frac{K_{O L}}{\omega_{3}}
$$

Hence, the proportional gain of the controller is expressed as:

$$
K_{p}=\frac{1+35.5 K_{t} K_{O L N}}{251.15}
$$

\section{RESULTS AND DISCUSSION}

The control algorithm was tested in Matlab and Simulink environment and Fig.4 indicates the synoptic scheme of the tape drive system control with PI controller. Also, the proportional-integral (PI) controller parameters and the corresponding system characteristics were obtained from Matlab script. An $\mathrm{m}$-file function based on the derived equations (1) to (29) is developed in Matlab environment. Similarly, the values of the proportional gain are computed based on equation (30) and the simulated value of the open loop gain $\left(\mathrm{k}_{\mathrm{lon}}\right)$. In this study, eight different values of $\mathrm{k}_{\text {oln }}$ are considered with eight iterations to determine appropriate controller parameters that would meet the system requirement. The electrical constant of the motor and other parameters were extracted from the datasheet. Table 1 shows the parameter values of the tape drive system and a Simulink model in fig.5 has been designed and implemented in order to simulate the tape drive system.

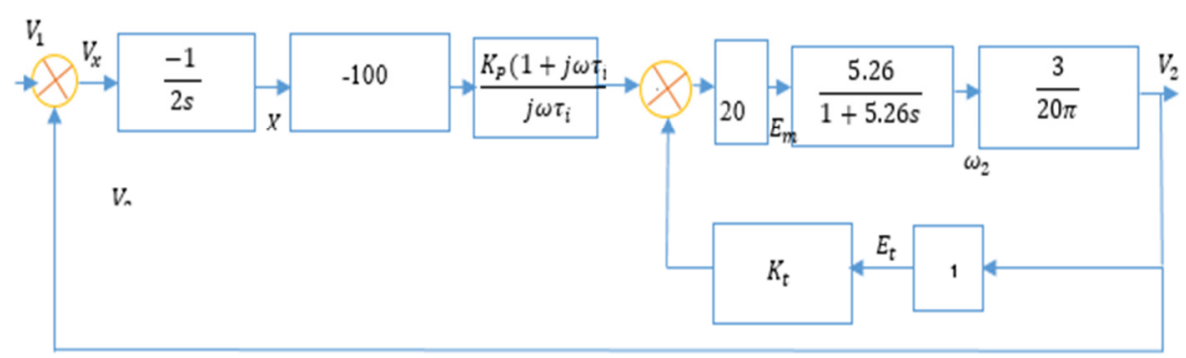

Fig. 4: s-domain block diagram of tape drive system 


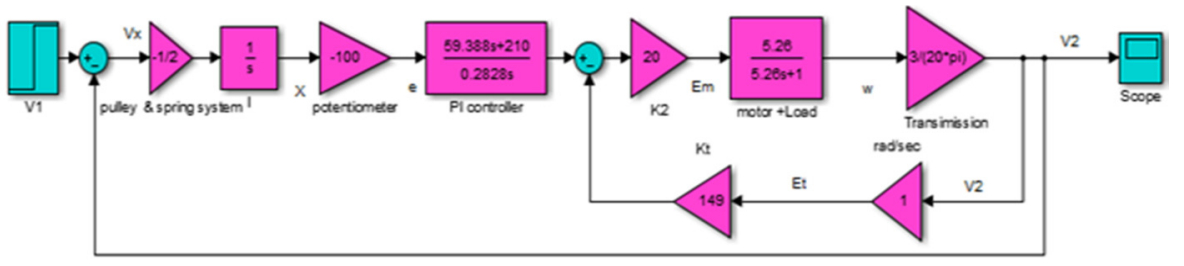

\begin{tabular}{|c|c|c|c|c|c|c|c|c|c|}
\hline \multicolumn{10}{|c|}{$\begin{array}{l}\text { Fig.5: Simulink model of the overall tape drive system } \\
\text { Table 1. Parameters of tape drive system }\end{array}$} \\
\hline parameter & $\begin{array}{l}J m \\
\left(\mathrm{kgm}^{2}\right)\end{array}$ & $\begin{array}{l}\text { Tstall } \\
(-)\end{array}$ & $\begin{array}{l}e_{a} \\
(v)\end{array}$ & $\begin{array}{l}\mathrm{N}_{\text {no-load }} \\
(\mathrm{rev} / \mathrm{min})\end{array}$ & $\begin{array}{l}\mathrm{K}_{2} \\
(-)\end{array}$ & $\begin{array}{l}K_{s} \\
(N / m)\end{array}$ & $\begin{array}{l}\mathrm{C}_{\mathrm{p}} \\
(\mathrm{v} / \mathrm{m})\end{array}$ & $\begin{array}{l}V_{2} \\
(m / s)\end{array}$ & $\begin{array}{l}E_{t} \\
(V)\end{array}$ \\
\hline value & 0.1 & 2.0 & 20 & 1000 & 20 & 10 & 100 & 5 & 5 \\
\hline
\end{tabular}

The input velocity is set to $1 \mathrm{~m} / \mathrm{s}$ and to obtain the desired velocity at the output, a proportional-integral feedback strategy is implemented such that the system requirement is achievable. In the light of this, various open loop gains are implemented and tested to ensure that the system design requirement is attainable irrespective of the discrepancies in the velocity. Fig.6. shows the step responses of the velocity at $\mathrm{k}_{\text {oln }}$ is $1,5,7.07$ and 8 .

The curves indicate that at $\mathrm{k}_{\mathrm{oln}}$ equal 1 and 5, the system is overdamped, this means that system has real poles at the left half plane. However, the values of the proportional gain corresponding to the open loop gain are not sufficient to minimize the velocity tracking error, hence, the design specifications are not met as shown in table.2. similarly, the curves when koln is 7.07 and 8 show $7.31 \%$ and $7.71 \%$ overshoot, $1.08 \mathrm{sec}$ and $1.07 \mathrm{sec}$ peak time, $0.385 \mathrm{sec}$ and $0.346 \mathrm{sec}$ settling time, $67.2 \mathrm{deg}$. and $65.6 \mathrm{deg}$. phase margins and 149 and 168 proportional gain respectively. It can be deduced from the curve that the controller parameters sufficiently meet the system requirement and at these values, the velocity output is $1 \mathrm{~m} / \mathrm{s}$ which indicates the controller minimize the error as close as zero.
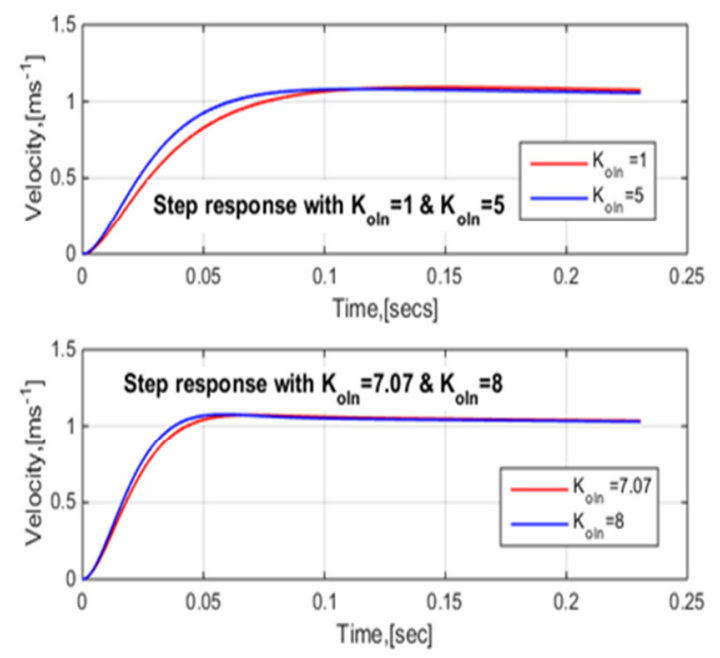

Fig.6: step response at $\mathrm{K}_{\mathrm{oln}}=1,5,7.07 \& 8$
Also, Fig.7 indicate the velocity responses at the values of $\mathrm{k}_{\text {oln }}$ equal 10 and 15 . The curve at koln equal to 10 has the following transient characteristics $9.41 \%$ overshoot, $1.09 \mathrm{sec}$ peak time, $0.281 \mathrm{sec}, 210$ proportional gain and $62.3 \mathrm{deg}$. Phase margin.
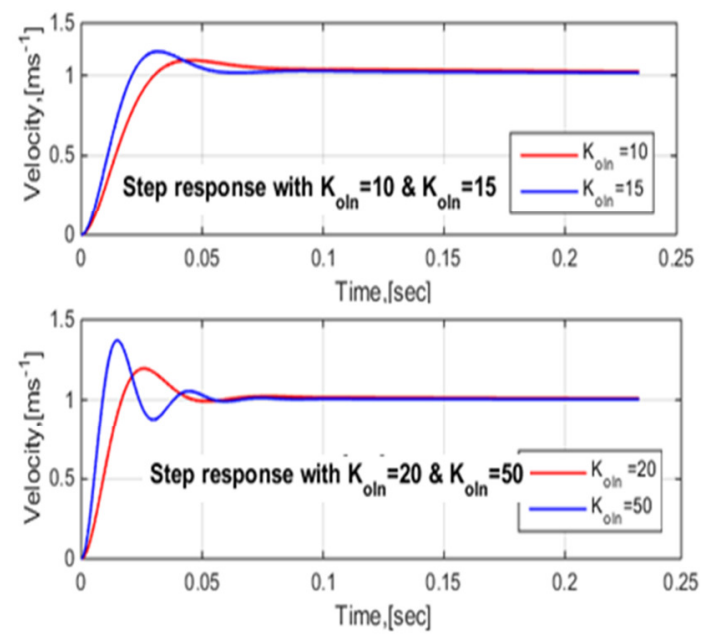

Fig.7: step response at Koln $=10,15,20, \& 50$

These characteristics show that the controller is capable of tracking the input velocity with little error with a well-damped response without oscillation. However, other values of $\mathrm{k}_{\text {oln }}$ are not suitable parameters to track the input velocity of the tape drive system. Notably, the curves at 15, 20 and 50 value of $k_{\text {oln }}$ show sign of system oscillation due to the high overshoot experience as a result of the location of the conjugate poles. Furthermore, in Fig. 8 the step response for the eight cases of open loop gain is superimposed for comparison. It is noted from the graph that the response at 7.07,8 and 10 values of $\mathrm{k}_{\text {on }}$ show a critically damped system with the fastest response. Fig.9 and 10 show the open loop and closed loop bode plot respectively with corresponding phase margin as indicated in Table2. 


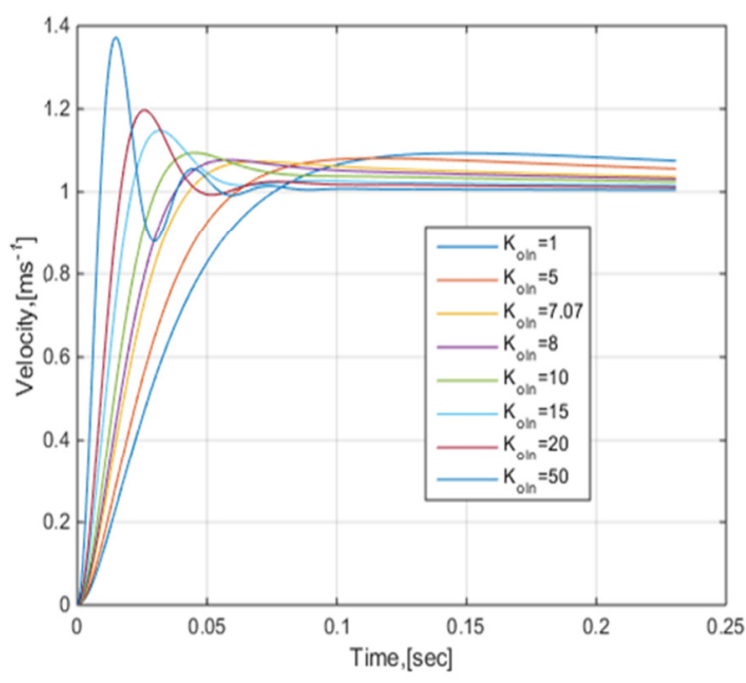

Fig.8: step response with all values of Koln

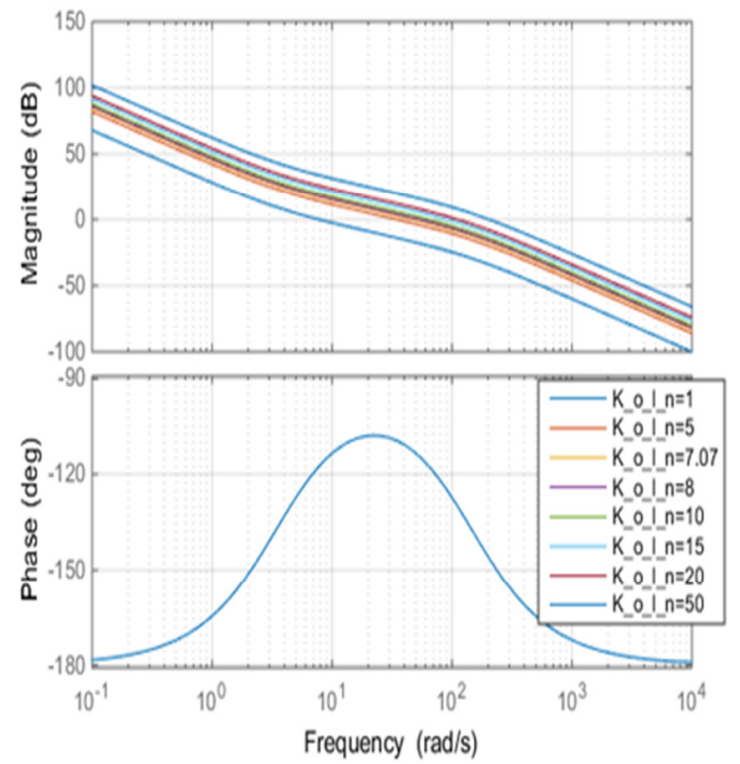

Fig.9:open loop bode plot at Koln=1, 5,7.07,8,10,15,20 \& 50

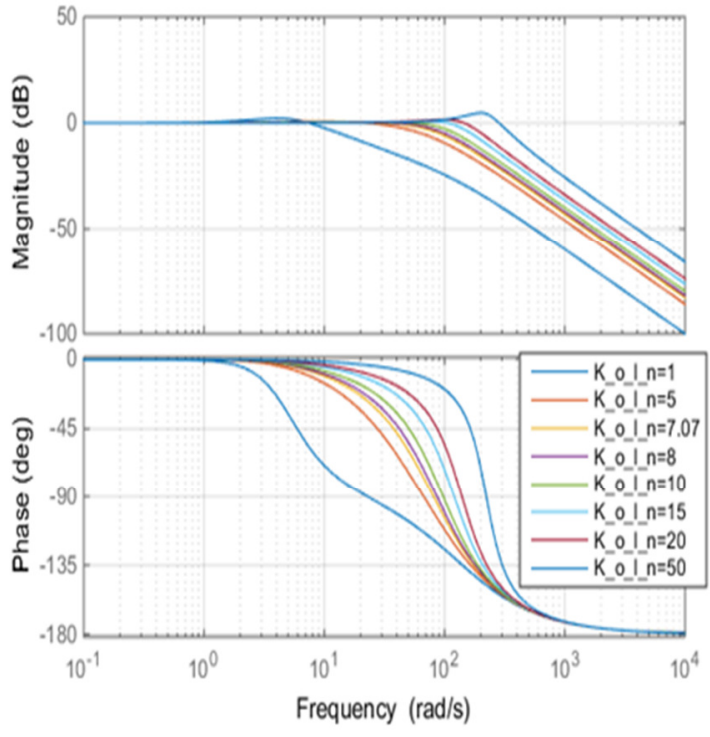

Fig.10: closed loop bode plot at $K_{\text {oln }}=1,5,7.07,8,10,15,20 \& 50$

Table 2.Predicted characteristics for the tape drive system

\begin{tabular}{lllllll}
\hline$K_{\text {oln }}$ & $\begin{array}{l}\% \\
\text { OS }\end{array}$ & $\begin{array}{l}\text { Tp } \\
(\mathrm{sec})\end{array}$ & $\begin{array}{l}T s \\
(\mathrm{sec})\end{array}$ & $\tau_{i}(-)$ & $\begin{array}{l}K_{p} \\
(-)\end{array}$ & $\begin{array}{l}P . M \\
(\mathrm{deg})\end{array}$ \\
\hline 1 & 21.9 & 1.22 & 0.958 & 0.2828 & 21 & 62.4 \\
5 & 8.1 & 1.08 & 0.488 & 0.2828 & 105 & 70.4 \\
7.07 & 7.31 & 1.07 & 0.385 & 0.2828 & 150 & 67.2 \\
8 & 7.71 & 1.08 & 0.346 & 0.2828 & 168 & 65.6 \\
10 & 9.41 & 1.09 & 0.281 & 0.2828 & 210 & 62.3 \\
15 & 14.8 & 1.15 & 0.161 & 0.2828 & 315 & 55.4 \\
20 & 19.7 & 1.2 & 0.052 & 0.2828 & 421 & 50.0 \\
50 & 37.2 & 1.37 & 0.091 & 0.2828 & 1052 & 33.9 \\
\hline
\end{tabular}

Conclusions: This paper presents a velocity tracking control for tape drive system used for data storage. In this study, mathematical model was formulated based on physical laws that governing the model subsystems and subsequently converted to the frequency domain for accurate simulation of the system's dynamics. Simulation results show that the controller performance depends on the numerical value of $\mathrm{k}_{\mathrm{oln}}$ and larger open loop gain resulted in worst controller performance. Robust control schemes such as an adaptive controller and state feedback controller should be focus of interest in further research of tape drive system control.

Acknowledgements: The authors wish to acknowledge the kind support of HAN University of Applied Sciences the Netherlands and Olabisi Onabanjo University for providing facilities and support.

\section{REFERENCES}

Biskeborn, RG; Fontana, RE., Lo, CS; Czarnecki, WS; Liang, J; Iben, IET; Hipolito, VA. (2018). TMR tape drive for a 15 TB cartridge. AIP Advances, 8(5), 56511. 
Boettcher, U., Raeymaekers, B., de Callafon, R. A., \& Talke, F. E. (2009). Dynamic Modeling and Control of a Piezo-Electric Dual-Stage Tape Servo Actuator. IEEE Transactions on Magnetics, 45(7), 3017-3024.

Cherubini, G., Furrer, S., \& Lantz, M. A. (2016). High-Rate Skew Estimation for Tape Systems. IFACPapersOnLine, 49(21), 7-12.

Cherubini, G., Pantazi, A., \& Lantz, M. (2016). Nearoptimal tape transport control with feedback of velocity and tension. IFAC-PapersOnLine, 49(21), 19-25.

de Callafon, R. A., \& Wang, L. (2013). Adaptive Regulation of Time Varying Disturbances in a Tape Storage System*. IFAC Proceedings Volumes, 46(5), 478-483.

Garifi, K., Pao, L., \& Touri, B. (2017). Model Predictive Control for Track Following and Disturbance Rejection in a Tape Drive System * *This work was supported in part by the US National Science Foundation (NSF Grant CMMI-1234980), and the Hanse-Wissenschaftskolleg Institute for Advanced Study. IFAC-PapersOnLine, 50(1), 10864-10869.

Kim, M., Gentilini, I., \& Messner, W. (2010). Active Tape Edge Position Control System Using a Complex Proportional-Integral-Lead Compensator. In System (pp. 2653-2658).

Panda, S. P., \& Engelmann, A. P. (2003). Control and operation of reel-to-reel tape drives without tension transducer. Microsystem Technologies, 10(1), 53-59.

Panda, S. P., \& Laverenz, D. J. (2003). Modeling, control, and operation of threader mechanism in tape drives. Microsystem Technologies, 10(1), 60-65.

Pantazi, A., Cherubini, G., Ogura, E., \& Jelitto, J. (2014). Tape transport control based on sensor fusion. IFAC Proceedings Volumes (Vol. 47). IFAC.

Pantazi, A., Jelitto, J., Bui, N., \& Eleftheriou, E. (2010). Track-follow control for tape storage. IFAC Proceedings Volumes, 43(18), 532-537.

Pantazi, A., Jelitto, J., Bui, N., \& Eleftheriou, E. (2012). Track-following in tape storage: Lateral tape motion and control. Mechatronics, 22(3), 361-367.

Pantazi, A., \& Lantz, M. A. (2014). Tape drive track following using cascade control. IFAC Proceedings Volumes, 47(3), 5896-5901.

Zhong, H., \& Pao, L. Y. (2011). Combined Feedforward/Feedback Control for Tape Head TrackFollowing Servo Systems. IFAC Proceedings Volumes, 44(1), 4040-4045. 